\title{
Impact of Human Diffusion and Spatial Heterogeneity on Transmission Dynamics of Mosquito-borne Diseases
}

\author{
Cynthia Mui Lian Kon and Jane Labadin \\ Department of Computational Science and Mathematics \\ Faculty of Computer Science and Information Technology \\ Universiti Malaysia Sarawak, Kota Samarahan, Sarawak. \\ cynkonml@hotmail.com and ljane@fit.unimas.my
}

\begin{abstract}
Mosquito-borne diseases are of tremendous public health concern. To study and enhance the understanding of these infectious diseases, we present a reaction-diffusion generic model for mosquito-borne diseases. It is significant that a model can be generalized for mosquito-borne diseases. One of the objectives of mathematical models is to identify factors which contribute to the spread of diseases. The traveling wave front is examined and the minimum spread speed is acquired numerically. We analyze the impact of human random movement and spatial heterogeneity on the dissemination of disease through numerical simulations. It is shown that the increment of human diffusion decreases the basic reproduction number. However, spatial heterogeneity in transmission of disease contributes to the upsurge of infection.
\end{abstract}

Keywords - diffusion, generic model, mosquito-borne diseases, transmission, spatial heterogeneity

\section{INTRODUCTION}

Mosquitoes spread pathogen and parasites from one human to another human or animal through their bites. The common mosquito-borne diseases are dengue, yellow fever, chikugunya, encephalitides and malaria. According to the World Health Organization (WHO), 1.4 billion of the South-East Asia region's population is at risk of contracting malaria [1]. It is also reported that there were 2.4 billion dengue cases from 2000 to 2011 [2].

Mathematical models are advantageous to predict future outcome of diseases and to investigate and determine the ideal control measure. Simple deterministic models make assumption that the environment is homogeneous. On the contrary, populations are distributed in space and individuals are mobile. Space can bring about changes in the dynamics of populations. Gause's experiments with paramecium and didnium indicated that space makes an impact as one or both species eventually extinct when they both can coexist in nature [14]. Diffusion models take into account the movements of individuals based on random walk. One way of studying the effects of space is through reaction-diffusion models. In 1951, Skellam developed a reaction-diffusion model of invasion biology [3]. He established the relationship between random walks as movement of individual members of biological species and the diffusion equation as a description of dispersal of the population as a whole [15]. A reaction-diffusion system for chemical basis of morphogenesis was constructed by Turing [4] and he discovered diffusion-driven instabilities. The spread of rabies among foxes were modeled and the progress of rabies if introduced near Southampton was carried out by Murray et al. [5]. The model suggests that vaccination is a much beneficial control strategy compare to culling. Eventually, Takahashi et al. took into account diffusion and advection of Aedes aegypti and studied the existence of travelling wave in different situations and the minimum spread speed which corresponds to biological invasion [6]. Lewis et al. modeled the spatial spread of West Nile Virus based on a model by Wonham et al. [7]. They have found that the spread rate is an increasing function of the bird diffusion. A periodic Ross-Macdonald model which incorporates diffusion and advection was developed and analyzed. It was found that the spreading speed depends on certain parameters of the model thus control strategies can be determined [8].

In this paper, a generic reaction-diffusion model for mosquito-borne diseases is introduced. After which, the traveling wave solution which connects the disease-free equilibrium and endemic equilibrium is scrutinized. The minimum spread speed is suggested through numerical results. Furthermore, the effect of human mobility and spatial heterogeneity on the transmission of the disease is analyzed numerically. Finally, the conclusion and future work of this research are presented.

\section{FORMULATION OF MODEL}

As we want to develop a mathematical model which accommodates multiple mosquito-borne diseases, a study was carried out on mathematical models for vector-borne diseases and similarities were distinguished as mentioned in [16]. First of all, the compartments used were compared and it was found that the common compartments are Susceptible and Infectious for both human and mosquito population. Hence, the generic mosquito-borne diseases model [9] consists of SusceptibleInfectious (SI) compartments for both host and vector. Immunity and superinfection are not taken into account here as we want the model to apply to most mosquito-borne diseases 